\title{
The Evolution of Laser in Laryngology
}

\author{
Asil Tahir \\ Department of Otolaryngology/Head \& Neck Surgery, Leighton Hospital, Crewe, UK \\ Email: asiltahir@hotmail.com
}

Received 20 February 2015; accepted 13 March 2015; published 17 March 2015

Copyright (C) 2015 by author and Scientific Research Publishing Inc. This work is licensed under the Creative Commons Attribution International License (CC BY). http://creativecommons.org/licenses/by/4.0/

c) (7) Open Access

\begin{abstract}
Technological breakthroughs in physics are often adapted and incorporated into the ever growing field of otolaryngology. When first discovered, "The Incredible Laser" had promised to be science's new "Aladdin's lamp", it can "light up the moon", "kill instantly", or "perform miracle surgery" [1]. Although not quite fulfilling these roles, laser technology has been a key element in the development of endolaryngeal surgery. This article looks at the invention of Laser and it's progression into an invaluable tool in the field of laryngeal surgery.
\end{abstract}

\section{Keywords}

\section{Laser, History, Laryngology}

\section{The Discovery of Laser}

Albert Einstein propelled the theoretical foundation for the development of this landmark invention in 1916 when he proposed that photons could stimulate emission of identical photons from excited atoms [1]. Stimulated emission is where a photon interacts with an excited molecule or atom and causes the emission of a second photon having the same frequency, phase, polarization and direction. This theory has been fundamental in the development of laser technology. In 1940, the Russian physicist Valentin Fabrikant proposed that stimulated emission in a gas discharge may amplify light under specific conditions [1].

However, it was not until after the end of World War II when research into laser technology benefitted greatly from corporate and government investments into technological progress during the cold war era. This coupled with the growth in numbers of physicists and engineers and growth in the economy created the foundations for the invention of the ruby laser in 1960 by Theodore Maiman [2]. Soon after Maiman built the first laser, his assistant joked that the laser was "a solution looking for a problem." This of course contained some truth to it, as the laser was not a device invented to fill specific application requirement. It was more a discovery than an invention, a way to generate coherent light that laser developers expected would find applications in broader areas, such as research or communications [1]. 


\section{The Principles of Laser Surgery}

The atoms of a laser exist within a medium which can be either solid, liquid or gas. This is encased within an optical resonant chamber in-between two mirrors. The process of laser emission begins with an external source of energy, such as a flash lamp or an electric arc, which is used to excite the atoms in the medium. The internal energy contributes to a cascade of stimulated emissions that create the amplified light energy. The three important properties to laser are the wavelength, coherence and directionality. The particular wavelength of laser energy will decide it's precision as a scalpel and the haemostatic properties. The laser energy must be intense, sharply focused, and absorbed almost entirely at the surface for precise dissection with minimal surrounding thermal injury and tissue damage [1] [3] [4].

Currently there are a large number of lasers available for the otolaryngologist to utilise. They have varying characteristics including different wavelengths, interactions with tissue, modes of transmission and delivery systems. These include the Carbon Dioxide $\left(\mathrm{CO}_{2}\right)$, neodymium:yttrium-aluminum-garnet (Nd:YAG) in contact and non-contact modes, argon, potassium titanyl phosphate (KTP), Pulsed Dyed Laser (PDL), argon dye, holmium:YAG, erbium:YAG and free electron lasers [3] [5].

Further to its growing use in laryngology, laser surgery has developed in other areas of otolaryngology. Tumours of the tongue, floor of the mouth, palate and tonsils can be resected with excellent control and haemostasis using the $\mathrm{CO}_{2}$ laser. The Nd:YAG, $\mathrm{CO}_{2}$, KTP, and diode lasers have all been used with good short-term results of nasal airway obstruction and recurrent epistaxis. In ear surgery the precision of argon, KTP, and $\mathrm{CO}_{2}$ lasers through a microscope have been shown to be very effective in stapedotomy, ossicular fixation and tympanosclerosis [3] [6].

Laser surgery has advantages when compared to cold surgery on the larynx. It offers an unobstructed view of the operation field, ability to work at longer distances, minimises tissue manipulation, increased sterility, better haemostasis and is associated with fewer post operative complications. However, lasers require a large number of personnel to ensure effectiveness and safety. There is also a significant cost associated with the installation of equipment, maintenance and updating technical developments can be significantly more costly than using cold instruments. Laser heat can increase scarring and cause damage to adjacent tissue. Other potential limitations of laser include potential for endotracheal explosion, facial burns, mucosal burns, vocal fold webs, stenoses and glottic incompetence [3]-[5].

\section{The Carbon Dioxide Laser}

It was not until 1964, when Patel invented the first carbon dioxide $\left(\mathrm{CO}_{2}\right)$ laser, did it gain immediate popularity and rapidly developed as an important surgical tool in laryngology [7]. The $\mathrm{CO}_{2}$ laser was the first to be used on the larynx due to its ideal tissue properties for surgery; water absorbs the 10,600 nm wavelength really well, its high focus limits soft tissue penetration and minimises collateral thermal damage whilst providing good haemostasis [8]. It was initially experimented on cadaveric larynx and dogs and only became medically effective with the development of coupling of the microscope and the development of the micromanipulators by Polanyi and Bredemeier to deliver precise energy to the larynx [9].

Hungarian born Geza Jako, often called father of Laser surgery and was Inventor over 120 instruments. He introduced both cold microlaryngeal instruments in 1962, and in 1972 Jako experimented with the $\mathrm{CO}_{2}$ laser integrated with a microscope on 12 dogs as a precise haemostatic scalpel on laryngeal tissue. He selectively evaporated predetermined amounts of normal vocal cord tissue through a laryngoscope with good results. All the dogs received antibiotics to reduce risk of complications post anaesthesia. One dog had died prior to follow up laryngoscopy and three dogs had accidental radiation to the trachea. The dogs were killed with anaesthesia overdose after a 3 - 4 weeks follow up laryngoscopy revealing normal healing similar to that of a clean surgical wound repair. The larynges were taken for histology and showed distinct demarcation between tissue destruction and normal tissue with good healing and epitheliazation [10]. $\mathrm{CO}_{2}$ laser was especially useful for the majority of surgeons as the delivery of joystick and pedal enhanced the manual dexterity of surgeon working at a distance under high magnification. He advocated that the $\mathrm{CO}_{2}$ laser should be used synergistically with cold instruments and not as an alternative [3].

\section{The Use of Laser on Patient's Larynges}

The first use of laser in patients was in 1975 by Strong who used the $\mathrm{CO}_{2}$ laser to successfully treat 11 patients 
with early T1 laryngeal Cancer [11]. He described inadequate exposure or extension of the carcinoma to the anterior commissure, arytenoid or subglottic areas was a contraindication to laser excision. The excised areas showed good precision and very good healing even in post radiation larynges. The post operative voice was also shown to be as good as the remaining larynx would allow [11].

Joining his colleagues Strong and Jako, Vaughan from Boston Massachusetts went on to investigate the useful application of using $\mathrm{CO}_{2}$ lasers in laryngeal cancers. They showed that the laser is a useful adjunct in treating laryngeal tumours, not only through direct excision, but also by helping establish proper staging, diagnosing reoccurrence after radiation therapy, debunking tumour mass for airway establishment and prior to radiotherapy and chemotherapy. This was all possible with minimal morbidity, as a day case, with good organ preservation and without requiring tracheostomies. All of which worked out well for cost benefit [12]. They also went on to explore its use with various benign and malignant disorders-including carcinoma, stenosis, papilloma, nodules, polyps, cysts and amyloidosis [13]. Nonetheless, their novel approach was met with biting resistance.

Building on these results Steiner developed new endoscopes, instruments and new techniques to handle the larynx and improve oncologic effectiveness [14]. Between 1979 and 1986 he treated almost 900 patients aged 15 91 including over 500 patients with laryngeal carcinoma. He describes that in early stages of growth, transpolar microsurgical resection using $\mathrm{CO}_{2}$ laser is curative. And also now with laser the possibility of treating advanced tumours with or without radiotherapy while preserving functionally important organs without the need for tracheostomy. However in whenever the carcinoma has spread beyond the larynx or into arytenoids the use of laser is very limited [14]. A more recent study on laser treatment presented by Wolfgang Steiner, MD, in 2005 showed that of 333 patients with T1a tumors, $89.3 \%$ had their lesions controlled after five years, and $97.6 \%$ of those had their larynx preserved [15].

\section{Photoangiolytic Lasers}

Although $\mathrm{CO}_{2}$ has initially been the primary laser used in Laryngology, fiber based photoangiolytic lasers such as Pulsed Dyed Laser (PDL) and potassium-titanyl-phosphate (KTP) are now increasingly used on laryngeal pathologies. Unlike the $\mathrm{CO}_{2}$ laser the PDL and KTP deliver energy through thin glass fibres better suited to treat subepithelial resection such as nodules, cysts and polyps [3].

In the 1980s Anderson and his colleagues began using PDL on benign laryngeal lesions. These lasers have been previously specifically useful at treating vascular lesions on skin dues to the its wavelength which targets the absorbance peak of oxyhaemaglobin [16]. These were shown to be significantly effective on papillomatosis and dysplasia. The epithelium was largely preserved during laser resection, which meant these lesions could be treated without the concern of scarring, synechia or webbing. This was especially useful for lesions affecting the anterior commissure, which was commonly associated with an increased morbidity with repeat resection using cold instruments or $\mathrm{CO}_{2}$ laser.

PDL and KTP lasers have recently been used to treat laryngeal cancers by targeting their blood supply and also in the management of Reinke's oedema, which would conventionally require cold knife surgical intervention [17]. Although significantly more expensive that $\mathrm{CO}_{2}$ lasers, $\mathrm{PDL}$ has the potential of being carried out in a clinical setting using a flexible endoscope under local anaesthetic. This approach is likely to expand rapidly due to a reduction in patient morbidity and the potential of improvement in health-care delivery [3].

\section{The Future}

Years of development have vastly improved laser performance and have been an important solution for a variety of problems, not only in laryngology, but also in scientific research, consumer products, telecommunications, engineering and a host of other applications.

In the treatment of laryngeal cancer, laser surgery has already demonstrated reliable tumour removal with fewer complications than open surgery in managing many glottic and supraglottic lesions. Currently, there are a variety of different lasers available for otolaryngologists to utilise with extended applications and variable wavelengths such as $\mathrm{CO}_{2}$, Nd:YAG, argon, PDL, KTP and argon dye. New lasers, such as the use of thulium on endolaryngeal resections, show promising results offering improved haemostasis and effective tangential dissection. The increased volume of ambulatory and office-based laser surgery has an important role in reducing costs and will continue to gain increased interest in financially stretched healthcare systems around the world. The considerable amount of research that continues to develop the use of laser in otolaryngology inevitably means 
further advancements in its clinical application [3] [4].

\section{Conflicts of Interest}

I can confirm there is no financial or personal relationship with any people or organizations related to this report that could inappropriately influence this work.

\section{References}

[1] Hecht, J. (2010) A Short History of Laser Development. Applied Optics, 49, F99-F122. http://dx.doi.org/10.1364/AO.49.000F99

[2] Maiman, T.H. (1960) Stimulated Optical Radiation in Ruby. Nature, 187, 493-494. http://dx.doi.org/10.1038/187493a0

[3] Zeitels, S.M. and Burns, J.A. (2006) Laser Applications in Laryngology: Past, Present, and Future. Otolaryngologic Clinics of North America, 39, 159-172. http://dx.doi.org/10.1016/j.otc.2005.10.001

[4] Yan, Y., et al. (2010) Use of Lasers in Laryngeal Surgery. Journal of Voice, 24, 102-109. http://dx.doi.org/10.1016/j.jvoice.2008.09.006

[5] Shapiro, J., Zeitels, S.M. and Fried, M.P. (1992) Laser Surgery for Laryngeal Cancer. Operative Techniques in Otolaryngology—Head and Neck Surgery, 3, 84-92. http://dx.doi.org/10.1016/S1043-1810(10)80245-3

[6] Ossoff, R.H., et al. (1994) Clinical Applications of Lasers in Otolaryngology-Head and Neck Surgery. Lasers in Surgery and Medicine, 15, 217-248. http://dx.doi.org/10.1002/lsm.1900150302

[7] Patel, C.K.N. (1964) Continuous-Wave Laser Action on Vibrational-Rotational Transitions of $\mathrm{CO}_{2}$. Physical Review, 136, A1187-A1193. http://dx.doi.org/10.1103/PhysRev.136.A1187

[8] Rubinstein, M. and Armstrong, W.B. (2011) Transoral Laser Microsurgery for Laryngeal Cancer: A Primer and Review of Laser Dosimetry. Lasers in Medical Science, 26, 113-124. http://dx.doi.org/10.1007/s10103-010-0834-5

[9] Polanyi, T.G., Bredemeier, H.C. and Davis Jr., T.W. (1970) $\mathrm{A} \mathrm{CO}_{2}$ Laser for Surgical Research. Medical \& Biological Engineering, 8, 541-548. http://dx.doi.org/10.1007/BF02478228

[10] Jako, G.J. (1972) Laser Surgery of the Vocal Cords. An Experimental Study with Carbon Dioxide Lasers on Dogs. Laryngoscope, 82, 2204-2216. http://dx.doi.org/10.1288/00005537-197212000-00009

[11] Strong, M.S. (1975) Laser Excision of Carcinoma of the Larynx. Laryngoscope, 85, 1286-1289. http://dx.doi.org/10.1288/00005537-197508000-00003

[12] Vaughan, C.W. (1978) Transoral Laryngeal Surgery Using the $\mathrm{CO}_{2}$ Laser: Laboratory Experiments and Clinical Experience. Laryngoscope, 88, 1399-1420.

[13] Strong, M.S., et al. (1973) Laser Surgery in the Aerodigestive Tract. American Journal of Surgery, 126, 529-533. http://dx.doi.org/10.1016/S0002-9610(73)80044-3

[14] Steiner, W. (1988) Experience in Endoscopic Laser Surgery of Malignant Tumours of the Upper Aero-Digestive Tract. Advances in Oto-Rhino-Laryngology, 39, 135-144.

[15] Steiner, W., et al. (2004) Impact of Anterior Commissure Involvement on Local Control of Early Glottic Carcinoma Treated by Laser Microresection. Laryngoscope, 114, 1485-1491. http://dx.doi.org/10.1097/00005537-200408000-00031

[16] Anderson, R.R., Jaenicke, K.F. and Parrish, J.A. (1983) Mechanisms of Selective Vascular Changes Caused by Dye Lasers. Lasers in Surgery and Medicine, 3, 211-215. http://dx.doi.org/10.1002/lsm.1900030303

[17] Franco Jr., R.A., et al. (2003) 585-nm Pulsed Dye Laser Treatment of Glottal Dysplasia. Annals of Otology, Rhinology Laryngology, 112, 751-758. http://dx.doi.org/10.1177/000348940311200902 